\title{
Istruzioni per l'immaginazione. Tra didascalia ed ecfrasi
}

\author{
Piermario Vescovo \\ Università Ca’ Foscari di Venezia \\ vescovo@unive.it
}

\section{Riassunto}

Nel quadro di un lavoro dedicato alla teoria del testo drammatico, nel rapporto tra istanza diegetica e istanza narrativa, il presente intervento si dedica alla didascalia intesa, più che come "istruzione" scenica, come descrizione ecfrastica, dunque una "istruzione per l'immaginazione" del lettore o, se portata sulla scena, dello spettarore che ascolta. Il quadro temporale riguarda soprattutto il linguaggio letterario e la sperimentazione teatrale del Novecento, tuttavia nel rapporto con una tradizione del "visibile" di lunga durata.

Parole chiave: ecfrasi; didascalia; istanza diegetica; istanza mimetica.

\section{Abstract. Instructions for the imagination. Between caption and ecphrasis}

In the context of a project dedicated to the theory of the dramatic text, in the relationship between diegetic and narrative instances, the present intervention focuses on the caption intended, rather than as a stage "instruction", as an ecphrastic description, therefore an "instruction for the reader" or, if brought onto the scene, of the spectator who listens. The temporal framework concerns above all the literary language and theatrical experimentation of the twentieth century, however in the relationship with a long-lasting tradition of the "visible".

Keywords: ecphrasis; caption; diegetic instance; mimetic instance. 
1. Queste brevi note si situano sulla traccia di ricerche che mi occupano da lunga data, e che dovrò quindi riassumere almeno brevemente perché esse risultino minimamente comprensibili, e la loro occasionalità appaia meno relativa in rapporto a un quadro di riferimento.

Anzitutto si tratta di questioni relative a un tentativo di offrire una complessiva teoria del testo drammatico, impresa per cui la strumentazione narratologica ha costituito per chi scrive negli ultimi quindici anni un riferimento principale, presumendo soprattutto di indicare nel rapporto tra istanza mimetica (o drammatica) ed istanza diegetica (o narrativa) il nucleo essenziale, in un tentativo di comprensione esattamente contrario alle facili giustapposizioni di un "modello del testo teatrale" a un "modello del testo narrativo". Per essere più chiari, ciò significa il sostanziale rifiuto di quella strumentazione semiotica o semiologica che ha dominato il campo degli studi negli anni Settanta e Ottanta del secolo scorso, ovvero di quelli che sono stati anche i miei "anni di formazione", in favore di un ordine che si potrebbe senz'altro definire semantico, nell'idea o nel progetto di un "discorso del teatro" (nel senso del "discorso del racconto", più chiaramente nell'originale discours du récit, di Gérard Genette) e con un preciso riferimento, appunto per ciò che concerne l'opposizione di semantica a semiologia, al magistero di Émile Benveniste (Vescovo, 2007, 2011).

L'applicazione delle "figure" genettiane al teatro (al testo drammatico e più generalmente allo spettacolo) mentre permette, da una parte, di dare una precisa concretezza alle categorie del tempo (tempo dell'orologio contro dimensione cartacea del libro), significa, dall'altra e più in generale, la puntuale applicabilità anche di quelle "figure" che rappresentano l'istanza narrativa, nella rivendicazione, di contro alle affermazioni dello stesso Genette che sostengono "la duttilità temporale del racconto non avere equivalente sulla scena", dove "[nel]la rappresentazione [...] tutto è al presente per definizione". ${ }^{1} \mathrm{Ma}$, appunto, come la "lettura", più ancora che la disposizione testuale, può saltare, retrocedere, anticipare, ripetere rispetto alla continuità sintagmatica del testo, più ancora che della "storia", la linearità cronologica può essere manipolata tanto nel "racconto" quanto nella "rappresentazione". Come abbiamo documentato con esempi per ciascuna delle "figure" dichiarate da Genette per questo impraticabili, il teatro può permettersi invece di condensare e dilatare, oltre che ovviamente di tagliare sottoponendo ad "ellissi" parti o porzioni del tempo della "storia" nella rappresentazione (figure della durata), di ordinare diversamente nel tempo della rappresentazione le sequenze che sono irreversibili nella continuità del tempo della vita (figure dell'ordine), di mostrare più volte lo stesso episodio o di sommarizzare più episodi in uno (figure della frequenza singolativa e iterativa), e così via, nelle più complesse intromissioni dell'istanza narrativa nella mimesi drammatica (dominio che riguarda le categorie del modo e della voce). In tale quadro gli interessi teorici e il tentativo di una descrizione del sistema (a cui ho dedicato due libri) si sono

1. Rinvio alla "postilla teorica" in Vescovo (2011, pp. 195-223). 
completati con applicazioni specificamente storiche in un terzo libro, dedicato alla tripartizione modale, di origine platonica, nei secoli dall'età tardoantica all'età moderna, tra modo o genere narrativo o diegetico, modo o genere drammatico o attivo e, soprattutto, il dominante, anche per la drammaturgia, modo o genere misto (Vescovo, 2015b).

Conta qui ricordare soprattutto, oltre alle mie cose, il percorso di Silvia De Min, che ha dedicato un primo studio - inizialmente la sua tesi di laurea, discussa col sottoscritto - alla didascalia teatrale (De Min, 2013), e quindi, con due altri saggi, ha direttamente affrontato la questione del ruolo teatrale dell'ecfrasi, in particolare proponendo la categoria di "ecfrasi performata", in una formulazione in cui un aggettivo molto abusato, e in particolare negli studi teatrali degli ultimi decenni, trova una diversa appropriatezza nel senso della realizzazione scenica della "descrizione", anche e soprattutto nella riconsiderazione della didascalia e del suo ufficio (De Min, 2016a, 2018). Col primo dei tre libri qui ricordati De Min offriva in un campo come quello dello studio della didascalia - sostanzialmente viziato da due premesse date per scontate, ma in realtà largamente approssimative - un principio di osservazione completamente differente. Il primo luogo comune considera, infatti, la didascalia e il suo enorme sviluppo moderno, dalla seconda metà del XIX secolo in avanti, come un riflesso della regìa nella scrittura drammaturgica, e inquadra dunque come istruzioni sceniche quelle che (naturalmente eccettuati casi particolari: si vedano le esattissime didascalie di Beckett) sono spesso - e soprattutto presso alcuni grandi drammaturghi del Novecento (Pirandello, Valle-Inclán o Lorca, per citare solo esempi massimamente rappresentativi) - vere e proprie inserzioni narrative o liriche, di fatto inscenificabili (e, appunto, eventualmente solo eseguibili in forma di lettura o di proiezione del testo). Il secondo luogo comune degli studi dedicati alla didascalia riguarda invece un massiccio ricorso alla strumentazione semiotica, con l'esito di una catalogazione o distinzione di tipologie scarsamente significativa sotto il profilo ermeneutico, anche se certo qui più legittima, di fronte a un campo più ristretto e meno evanescente rispetto a quello generale, della cosiddetta "teatralità" (secondo una fortunata ma insufficiente definizione di Roland Barthes, da intendersi quale designazione del teatro al di fuori del testo, come se tutto il teatro dovesse riferirsi a un testo, che si risolve con l'indicazione dei "differenti codici" che presiedono alla spettacolo, ma con un'affermazione di principio che difficilmente passa dall'ovvia constatazione a un'analisi di dettaglio). Tra le idee più forti del libro di De Min si dà, indubbiamente, l'osservazione nell'impiego della didascalia presso i grandi autori del Novecento, ma non solo, della presenza e del peso in essa dell'istanza diegetica, da cui discendono vari corollari. Anzitutto nel fatto che il presunto statuto oggettivo della didascalia, che la vuole appunto descrizione di ciò che si deve o dovrebbe "far vedere" (e sentire) oggettivamente allo spettatore, risulta contraddetto dal forte impiego in essa dei principi di focalizzazione o prospettiva (ciò che risponde alla domanda genettiana: chi vede?, e con l'evidenza del fatto che in esse sono spesso l'autore o i personaggi, e non solo lo spettatore ideale, a vedere e sentire). La continuazione di queste 
interrogazioni sul campo più generale dell'ecfrasi, come si è appena ricordato, si esprime nel tentativo di una definizione teorica e storica di una precisa zona di questo, definibile nel senso di un dominio teatrale di quella che si è detta "ecfrasi performata", che riguarda insieme la didascalia e il racconto dei personaggi che sostituisce la diretta rappresentazione dell'azione con la sua descrizione (si pensi, in particolare, alla tragedia, alle sue esclusioni, e ai fondamenti che troviamo espressi fin dall'Ars poetica di Orazio, a proposito dei «multaque tolles ex oculis», nella raccomandazione "ne pueros coram populo Medea trucidet», e quel che segue: vv.183ss.). In tale prospettiva, dunque, si pone anche la principale chiusura del cerchio teorico rispetto alla questione di partenza.

Dall'altra parte, tornando ai miei interessi, una continuità di attenzione e applicazione - di cui credo la mia bibliografia renda atto - riguarda il rapporto tra testo e immagine, ragione sostanziale che si pone alla base di una serie di "incursioni" nel campo della storia dell'arte. Se dovessi riassumere sinteticamente il mio punto di vista o di metodo potrei dichiarare senz'altro una sostanziale estraneità al principio o alla tradizione dominante le applicazioni degli studiosi di letteratura che si interessano a temi e soggetti artistici, ovvero alla fortunatissima formula (ancora oraziana, tra l'altro!) dell'ut pictura poesis, laddove la tradizione occidentale si mostra viceversa definita dalla volontà $o$ dal cimento degli scrittori, e dunque anche dei drammaturghi, di "dipingere" attraverso la parola, imitando i "pittori" e le arti plastiche in genere. Il genere dell'ecfrasi si identifica strettamente con tale tradizione, tanto in una forma o destinazione esplicita, nel senso della categoria propriamente intesa (la finzione di opere d'arte, descritte attraverso la parola, dalle pareti del tempio di Giunone a Cartagine ai bassorilievi delle balze della montagna del Purgatorio, in opere di narrazione, ovvero le raccolte da Filostrato a Marino, per implicare con la Galeria di quest'ultimo anche una parola-chiave della codificazione moderna, dal secolo XVII in avanti), quanto in un'assunzione estesa, per ogni descrizione che si proponga la vivida restituzione dell'immagina attraverso la parola come a "farla vedere". Qui il teatro - e più precisamente il rapporto tra figurazione pittorica e figurazione teatrale ${ }^{2}$ - torna a mostrarsi campo di primario rilievo.

2. Passando dalla descrizione sommaria del campo di riferimento generale alla ricognizione specifica, vorrei richiamare l'attenzione su alcune righe di Gianfranco Contini che mi hanno, da molto tempo, colpito e che non ho mai avuto occasione di commentare.

Esse si leggono in uno dei suoi ultimi "esercizî" di applicazione, in un saggio tanto mirabile e denso quanto, sia permesso dirlo, discutibile, intitolato al Rinnovamento novecentesco del linguaggio letterario (1976; in Contini 1988, pp. 107-122). Entrare nel merito di queste pagine è impresa evidentemente

2. Il binomio e un ampio approccio nel capitolo di Ludovico Zorzi così intitolato (Zorzi, 1979, pp. 419-463). 
ardua e per più motivi sconsigliabile: troppi, infatti, i carichi per considerare le premesse e il significato di tale esempio in un quadro generale, e tuttavia, contemporaneamente, troppo alta la posta in gioco per rinunciare a un'almeno breve analisi, dove evidentemente essa non può non coinvolgere le questioni di fondo che invitavano lo studioso a produrlo.

Una breve osservazione, di estrema precisione, riguarda dunque in questo intervento uno scrittore che Contini amava pochissimo, ovvero Luigi Pirandello (antologizzato ne La Letteratura dell'Italia unita con una novella, La giara, con una considerazione ristretta non solo al narratore, ma al narratore collocato in una tradizione siciliana, che ne faceva in sostanza per Contini un epigono di Verga). Qui invece il fulminante giudizio riguarda il Pirandello maggiore, ovvero il drammaturgo, e una sintetica definizione dei caratteri essenziali della sua didascalia, ovvero della zona di intersezione della narrazione al testo drammatico. Contini ha ragionato - nelle righe precedenti - di una "forza maggiore" come "forma sottile", caratterizzante, e indice specifico di sperimentalità nella scrittura primo-novecentesca, quella del "frammento", che vedrebbe come suoi tratti salienti il primato della nominalità e della promozione per il tempo verbale dell'imperfetto in luogo del perfetto (si pensi, ovviamente, al D'Annunzio di Notturno e di ciò che ad esso segue). Ora Pirandello viene esattamente evocato come caso di sottrazione a tale "forza maggiore":

Formalmente, si ha una prova $e$ contrario nei casi di sottrazione alla forza maggiore, come nell'abbandono della nominalità nella didascalia teatrale di Pirandello, narrativizzata e sottomessa alla stessa sollecitazione espressiva (inclusione del gesto, deformazione iconica) che si ha nella generale oralità dell'autore (un'oralità monologante che, con tanta distanza di contenuti, trova un vero parallelo solo nel Palazzeschi della Piramide e altri scherzi; e qui andrebbe agganciato un lungo discorso sulla variabilità del soggetto referente, fino alla sua distruzione) (Contini, 1988, pp. 120-121).

Si tratta, con ogni evidenza, di un referto tanto più rilevante in quanto Contini, come abbiamo detto, non prova alcuna attrazione per o adesione alla scrittura di Pirandello, di contro all'atteggiamento che lo conduce in queste stesse pagine, $\mathrm{o}$ in altre prossime, ad indicare come riferimenti principalissimi nel "rinnovamento" del linguaggio letterario del Novecento non solo Céline, Joyce o Gadda, ma Roberto Longhi e Antonio Pizzuto (quest'ultimo si direbbe nel frattempo giustamente obliato, ove la sua marca pedantesco-burocratica è stata certamente sopravvalutata in senso stilistico di deformazione ed espressività). Il "rinnovamento" di cui qui si parla domina infatti un altro degli ultimi e principali saggi continiani, in cui lo studioso tentava, con dimensioni più ampie e in certo senso sistematiche, una ridefinizione stessa della categoria di espressionismo, che negli anni precedenti egli aveva definito e applicato in una direzione diversa, sostanzialmente di carattere linguistico. Mi riferisco alla voce Espressionismo letterario per l'Enciclopedia del Novecento (1977), dunque esattamente coeva all'intervento dedicato al rinnovamento del linguaggio letterario nel Novecento. (Contini, 1988, pp. 41-105). 
La constatazione relativa all'assenza nella didascalia di Pirandello della "forza maggiore" dell'uso dell'imperfetto, si potrebbe svolgere e ampliare proprio osservando ciò che un anche rapido spoglio di occorrenze offre, in una considerazione più ampia della questione della temporalità. Se il tempo dell'impressione dell'attimo fuggente e del divenire che si fissa nel frammento è l'imperfetto, qui appunto assente, tanto più significativa appare l'ambivalenza - o direi, poiché cronologicamente si tratta di un passaggio, come mostra la specifica variantistica tra redazioni - dal presente, per definizione didascalico e della descrizione, al futuro dell'ideale attuazione rappresentativa. ${ }^{3}$ Estraggo per l'univoco principio di intervento in questa direzione dall'intero testo di Sei personaggi in cerca d'autore, dalla prima alla seconda redazione, un unico esempio: "Ma già la scena tra la Figliastra e Madama Pace, durante la protesta degli Attori e la risposta del Padre, è cominciata piano, naturalmente, come non è possibile che avvenga su un palcoscenico" > "sarà cominciata ... come non sarebbe possibile": la revisione di questo passo offre tanto la correlazione del condizionale al futuro (di contro al doppio presente del testo di partenza), quanto l'indicazione di una realizzazione diversa da quella della scenificazione tradizionale, che si può intendere sia come altra (se si vuole qui una "diversa regìa") che come principio di immaginazione extrascenico offerto al lettore (Pirandello 1986-2007, vol. 2, II, p.1002). ${ }^{4}$

Non so valutare l'accostamento continiano del Pirandello didascalista al Palazzeschi della Piramide, che mi pare relativo se non incidentale (e diciamo pure di marca snobistica, dove il confronto va dal più noto al meno noto), ma certamente invece centrale risulta qui il riferimento alla "variabilità del soggetto referente, fino alla sua distruzione", che giunge al vero nucleo della scrittura pirandelliana, che non è certo nel cliché del rapporto tra "vita e forma" (che Ferdinando Taviani ha definito benissimo un mero slogan d'autore e poi del pirandellismo scolastico e divulgativo), ma in un totale nichilismo, di cui abbiamo recentemente provato a discutere riprendendo alcune brevi e illuminanti pagine di Furio Jesi. ${ }^{5}$ Che il "soggetto referente" (l'Autore, appunto, tra

3. Devo qui anche dichiarare un uso più ampio e insieme più caratterizzante e specifico del termine "rappresentazione", che infatti, seguendo la scelta ardita, operata dalla miglior edizione commentata moderna della Poetica (Dupont-Roc \& Lallot, 1980) impiego anche per mimesis, non reso dunque con "imitazione", secondo la traduzione piana e convenzionale (nel senso buono del termine). Basti qui - rispetto alle molte annotazioni offerte dal commento citato - riferire le ragioni generali di tale opzione dichiarate nelle pagine introduttive: «les connotations théâtrales de ce verbe et surtout la possibilité de donner pour complément, comme à mimeisthai, indifférement l'objet-"modèle" et l'objet produit - au lieu qu' "imiter" excluait ce dernier, le plus important - ne pouvaient qu'emporter la décision». Del resto anche parlando, per esempio, del romanzo sembra lecito ed utile dire che la descrizione di un personaggio è, più che "imitazione", "rappresentazione" (come mostra, appunto, la resa attraverso la parola anzitutto del suo aspetto fisico e della sua attitudine che tanto spazio ha anche nella tradizione didascalica moderna per il testo drammatico).

4. Si può facilmente scorrere per una ricognizione complessiva l'affiancamento su due colonne delle due redazioni di Sei personaggi.

5. Si veda Vescovo, 2020, pp.166-168 (Jesi: "quella di Pirandello è brutalità inutile senza 
l'altro quello di cui vanno in cerca gli abbozzi dei personaggi nel "brevetto" più ragguardevole e fortunato della storia del drammaturgo e del teatro italiano tutto della prima metà del Novecento) risulti anche nel sistema didascalico variabile, e variabile fino alla distruzione (o direi: all'annientamento), è referto esattissimo e che apre a considerazioni di portata assai ampia, che coinvolgono anche, fuori dallo spazio della didascalia e dell'immaginazione del teatro, l'oralità monologante che costituisce il tessuto del testo pirandelliano. Leggasi oralità sostanzialmente monologica, ivi compresa l'ossessiva, ampiamente articolata in senso di costruzione del periodo, presenza dei lunghi discorsi dei raisonneurs e del'autore che si fa con essi perenne postillatore di sé stesso, mentre effettivamente appartengono al registro didascalico (con vistosa contraddizione in termini rispetto all'etichetta) proprio la "inclusione del gesto" e la "deformazione iconica". Ciò che è impossibile "eseguire" leggendo la didascalia, perché di fatto inscenificabile, apre (oltre che a un orizzonte di discorsività intrusiva e commentativa dell'autore, in una definizione narratologica definibile nella figura della pausa) a un procedimento ecfrastico, nel senso esteso del termine. Si vedano descrizioni come le seguenti, che mi è capitato di citare spesso, dall'inizio de I giganti della montagna; scambiandole di ordine faccio precedere una di quelle riferite alla minuta rappresentazione, quasi pittorica, dei "caratteri" (con dettagli ovviamente irrestituibili nella caratterizzazione scenica) a uno dei luoghi più evidenti in cui la comparazione, peraltro per il piano auditivo, apre a prospettive impossibili nel senso di una realizzazione:

Tanto la Sgricia quanto Duccio sono scesi dalle loggette e ora son davanti la villa, sullo spiazzo erboso, costernati. Dalla porta appare Cotrone, ch'è un omone barbuto, dalla bella faccia aperta, con occhioni ridenti splendenti sereni, la bocca fresca, splendente anchéessa di denti sani tra il biondo caldo dei baffi e della barba non curati. Ha i piedi un po' molli e veste sbracato, un nero giacchettone a larghe falde e larghi calzoni chiari; in capo ha un vecchio fez da turco, e un po' aperta sul petto una camicia azzurrina.

Al levarsi della tela è quasi sera. Dall'interno della villa si ode, accompagnato da strani strumenti, un canto balzante, che ora scoppia in strilli improvvisi e or sabbandona in scivoli rischiosi, finché non si lascia attrarre quasi in un vortice, da cui tutt'un tratto si strappa mettendosi a fuggire come un cavallo aombrato. Questo canto deve dar l'impressione che si stia superando un pericolo, che non ci par l'ora che finisca, perché tutto torni tranquillo e al suo posto, come dopo certi momentacci di follia che alle volte ci prendono, non si sa perché.

Qui, peraltro, il referente, il cavallo aombrato, che non si sa come uno spettatore potrebbe immaginare ascoltando un'esecuzione del rumorista, risulta significativamente il punto d'approdo in termini visivi della descrizione auditiva.

mistificazione, poiché resa inutile, nihilistica ed essoterica fino in fondo della perdita di ogni passato collettivo, e perciò anche di ogni futuro e più che futuro"). 
3. Dall'esempio singolare al sistema, come si premetteva, secondo un percorso inevitabile, dove gli elementi-guida sono rappresentati da ciò che Contini considera - senza esemplificazioni - come "inclusione del gesto, deformazione iconica" nella didascalia pirandelliana, ovvero dimensione espressiva o senz'altro (secondo la riformulazione nel saggio dedicato alla categoria) espressionistica. Ho messo su carta queste note, e, anzi, ho deciso di riflettere sull'implicazione pirandelliana nel saggio di Contini, proprio contemporaneamente alla scrittura di un intervento pronunciato a un convegno per il centenario della nascita di Gianfranco Folena, dedicato alla questione del plurilinguismo e dell'espressionismo teatrale alla fine degli anni Settanta del secolo scorso.

Senza in quel caso voler entrare nella lunga e complessa gestione o gestazione continiana della categoria, mi sono potuto giovare dell'ottima, recentissima, ricostruzione di Luca D'Onghia della messa a punto dell'uso del termine (D’Onghia, 2020, pp. 79-96), che mi ha consentito tra l'altro, attraverso la documentazione di un impiego più vario e del suo divenire negli anni Sessanta, di leggere meglio il decisivo spostamento offerto dalla meditazione certo sistematica rappresentata dalla rammentata "voce" Espressionismo letterario per l'Enciclopedia del Novecento nel complessivo sistema continiano. Essa si apre, infatti, proprio con la distinzione tra una "ristretta originaria proprietà cronologica, geografica e intenzionale" del termine e una "portata corrente di valore polare, e per così dire categoriale" del medesimo (Contini, 1988, p. 41). La categoria - anche col nome di espressivismo - era servita a Contini, soprattutto nel celeberrimo saggio del 1963 che accompagnava l'edizione in volume della Cognizione del dolore di Gadda, a definire dal punto di arrivo una "funzione Gadda", nel tentativo di sdipanare il filo di un'ininterrotta tradizione fondante di "bilinguismo di poesia illustre e poesia comica" (con un punto di partenza collocato più indietro nel tempo rispetto a cui è lecito mettere in campo la categoria crociana di "letteratura dialettale riflessa", fino alle origini stesse della letteratura italiana). ${ }^{6}$ Il termine veniva dunque diversamente "polarizzato" nel corso del decennio successivo dallo studioso, in una definizione novecentesca ed europea, in cui quindi la questione del dialetto e del plurilinguismo si fa marginale o si ridefinisce in quella, appunto, diciamo più estesamente "linguistica", nel senso di formale o stilistica, e nell'ottica di quello che viene ritenuto un complessivo e sostanzialmente unitario (o unificabile) "rinnovamento" del linguaggio letterario, in cui dunque la stessa antichità di fondazione o la natura "costitutiva" della tradizione linguistica italiana perdono in intensità e valore.

Il campo di dette estensioni e applicazioni polari o categoriali, anche se con uno sguardo finale, nell'ultima pagina del saggio, alla tradizione italiana intera, in continuità alla precedente idea di un "bilinguismo costitutivo", risulta dunque in queste pagine interamente novecentesca, e non solo per i limiti imposti dalla sede a cui il contributo era destinato e per cui fu pensato. L'applicazione metaforica può sì richiamare nella pagina finale Quevedo, Rabelais o Jacopone

6. Anche su questo si vedano le attentissime osservazioni di Luca D’Onghia, e si veda il saggio parallelo che si cita più sotto (cfr. n. 11). 
(e addirittura Lucano!), ma secondariamente e nello spazio di un sommario riallargamento del campo, che voleva evidentemente comprendere la storia di quella categoria nelle riflessioni dell'autore. Si veda, infatti, come ho già provato a richiamare nell'intervento dedicato a Folena, nelle premesse di questa "voce", l'osservazione relativa al fatto che in tedesco, ovvero nella lingua in cui nacque la categoria, "mai l'espressionismo potrebbe avvicinarsi al significato di una semplice oltranza espressiva". Riferimento tanto più importante, posto che l'oltranza espressiva o la violenza al sistema non rappresentano affatto, evidentemente, il principio di scelta degli scrittori attraverso cui viene costruita la proposta del canone, non solo Céline o Joyce, come abbiamo già ricordato, ma anche Pessoa, e per l'Italia non solo Gadda, ma anche i "vociani", e Marinetti e Longhi e Pizzuto. Il "rinnovamento" novecentesco del linguaggio letterario vede, infatti, oltre che alcuni allargamenti attraverso definizione aggettivale (per esempio "espressionismo lirico"), la parallela inclusione, più che in opposizione a complemento, della categoria di "impressionismo", sempre dunque muovendo di fatto da esperienze di riformulazione dell'immagine nella parola e con una distinzione o polarizzazione delle due categorie in rapporto a una rispettiva prevalenza o dominante nominale o verbale.

Una "funzione Longhi", infatti, in luogo di una "funzione Gadda", domina tale tentativo di definizione, confidato a un saggio breve e legato a una contingenza (un intervento a un convegno, peraltro con un'ampia premessa di giustificazione di tale "occasione"), ma di fatto un equivalente formale dell'oggetto, o degli oggetti, di riferimento nel voler offrire in una sintesi ardua e breve una descrizione di ambizioni sistematiche e totalizzanti. A partire da esempi apparentemente sparsi e limitati si giunge dunque a una formulazione di ordine generale, che indica come fatto essenziale una "funzione del discorso rappresentativo" posta dentro a una funzione di "discorso referente". Espressionismo e impressionismo, categorie come si è già sottolineato di vistosa ed evidente mutuazione "artistica", dalla critica d'arte e dalla "prosa d'arte", sono dunque qui polarizzate e ridefinite nella rispettiva opposizione del verbale al nominale a definire tale "discorso rappresentativo", nella deroga moderna, si intuisce, a un regime di rappresentazione oggettiva.

E qui si potrà precisare meglio, almeno per questo più specifico uso $o$ polarizzazione, la questione della "discutibilità" a cui ho fatto, all'inizio di queste pagine, allusione: dichiarazione, che non potrà non suonare come ardita, da parte di chi intenda misurarsi con pagine tanto dense e difficili. In essa si inscrive anzitutto il dubbio sul rilievo e la pertinenza di alcuni di questi campioni, ma riguarda in particolare una mia personale distanza, e per certi versi "antipatia", da autori che invece caratterizzano in maniera forte l'identità stessa di un discorso letterario, e tanto più di una parte cospicua della critica italiana, e in particolare in rapporto al campo che abbiamo detto della storia dell'arte. Confesso, dunque, di non amare Roberto Longhi, vuoi per il suo discredito per alcuni pittori sommi (da Tintoretto a Tiepolo, ma si veda, di contro, anche la sua passione per uno scrittore come Marco Boschini che narrava come "messa in atto" la pittura di Tintoretto, tanto più significativa 
in rapporto al discredito dell'oggetto; cfr. Vescovo, 2015a, pp. 92-107), ma soprattutto, e non per colpa di Longhi, per il culto della sua scrittura anche presso alcuni grandissimi critici letterari del secondo e ultimo Novecento, e delle sue prosecuzioni/imitazioni fino al presente, con la conseguenza sulla prosa e sul "sistema di metodo" di tanta critica d'arte e storia dell' arte italiana.

In ogni caso, il saggio fissa in questo rapporto, dunque, una sorta di "funzione Longhi" a sostituire la precedente "funzione Gadda", passando da un impiego della categoria di espressionismo per il "bilinguismo" costitutivo della letteratura italiana, a quella del binomio di discorso "rappresentativo" e di "discorso referente". L'esempio dimostrativo viene infatti offerto da un passo in cui il critico maturo citava sé stesso giovane. Da qui la possibilità - pur trovando, ripeto, tutto questo particolarmente discutibile - di ripensare la questione di un "discorso dell'ecfrasi", da intendere in un'altra e più ampia accezione. Non la descrizione di un'opera d'arte immaginata e per cui, appunto, la parola gareggia con l'immagine che evoca, nel senso della tradizione dell' $u t$ poeta pictor, ma in cui lo stesso "atto critico" si pone sostanzialmente a partire da un'operazione di ricreazione dell'immagine - qui un'immagine reale, oggetto di analisi e commento - nel sistema della parola. L'immagine reale si fa in questa tradizione, per riprendere in altra posizione la definizione di Silvia De Min, "performata", nel senso che la descrizione la ricrea rispetto all'originale, e naturalmente la trasforma. Ciò permette, mi sembra, al contempo, una definizione più precisa e ristretta della categoria di "prosa d'arte", intendendola non nel senso diffuso e ampio del termine, ma in quello specifico che si definisce proprio nell'istanza della ricreazione verbale dell'immagine (l'importanza del "barocco" Marco Boschini - dotato di una lingua insieme tecnica ed espressiva, dove il veneziano offriva tanto il vocabolario tecnico delle "parole dell'arte" dei pittori veneti e della deformazione e reinvenzione dialettale veneziana nello specchio della tradizione letteraria italiana - è per questo assai rilevante per Longhi).

L'esempio cardinale - ovvero il referto più "discutibile" quale campione di "rinnovamento novecentesco del linguaggio poetico", ma indubbiamente tra tutti denso e rilevante - è offerto da Contini proprio da un passo di un Longhi che in un saggio del 1927 riprendeva le punte di "prosa d'arte" di sé stesso giovane, del 1914. Si tratta della descrizione della Vittoria di Costantino su Massenzio di Piero della Francesca (anzi, in dizione scelta e snobistica, di Piero dei Franceschi). Tra l'altro la ripresa risulta virgolettata non solo in quanto citazione, ma come una sorta di battuta pronunciata, appunto, da una voce implicata nel "discorso referente" (la voce del critico che si riprende come se si trattasse di parole di un personaggio o che sembrano provenire dallo stesso dipinto come "imperativo" che guidò la sua realizzazione). L'uso dell'imperativo, che tramuta la descrizione di ciò che si vede nel dipinto in dettato di "creazione", si potrebbe dire sostituisce il "performativo" del presente indicativo (nel senso degli speech acts dei linguisti) coll'assertivo, ovvero la modalità dell'enunciato che "descrive uno stato di cose esterno" (oggettivo e compiuto, 
come nel perfetto o "preterito epico", o in divenire e in movimento, come nel presente) con un "comando": 7

E, o voi, incorruttibili sfere di candido feltro, bilicatevi sul peltro degli elmi fino a che, nella luce abbacinata, ne diveniate sul petto cerulo del cielo, medaglie - di valore cromatico! (Contini, 1988, p. 113).

Ciò che un lettore appassionato di Longhi - compresi i grandi critici che hanno analizzato la sua "prosa" - trova elemento caratterizzante i suoi saggi maggiori (dove appunto la "traduzione" in "prosa d'arte" si suppone mezzo per la comprensione profonda dell'oggetto dell' analisi, in quanto "ricreato" in forma verbale), appare qui in una forma estrema (nel senso anche di eccessiva e ai limiti dell'autoparodia involontaria): che Contini selezionasse appositamente un Longhi "datato" e "giovanile", più "novecentesco" insomma, che abitava dentro al critico maturo, ci sembra tutt'altro che casuale.

4. In queste pagine, in entrambi i saggi citati, Contini nomina anche, rapidamente, Giovanni Testori, tra i "pregevoli prodotti epigonici" di Gadda; la "voce" Espressionismo letterario giunge meglio a ricordare, in un'altra rapida e parallela implicazione, anche i titoli di due allora recentissimi testi teatrali dello stesso, l' Ambleto e il Macbetto, definiti ancora come un episodio di sostanziale derivazione gaddiana.

Converrà a questo punto, e dopo le considerazioni che abbiamo appena condotto, ricordare precisamente il rapporto di Testori - del critico d'arte Testori del Sacro Monte di Varallo di Gaudenzio Ferrari (1965), anzi del "Gran teatro montano", suo discepolo, e poi dello scrittore - proprio con Roberto Longhi. L'Ambleto (1972), nell'incontro con un interprete ruzantiano quale Franco Parenti (e già compagno di Fo in imprese teatrali), metteva in realtà insieme le due questioni che abbiamo raccolto da Contini: quella del "discorso rappresentativo" (qui della "rappresentazione" propriamente intesa, nel senso teatrale del termine) e quella della tradizione dialettale e plurilinguistica, collocata sotto il cartello dell'espressionismo. Il ritorno di Testori al teatro si inquadra, e poteva già allora essere perfettamente compreso, nel panorama italiano del secondo e ultimo Novecento, in cui si tornava potentemente a guardare al dialetto e alle sue principali tradizioni, e alle stelle polari caratterizzanti, da Ruzante a Basile, nel crogiuolo caratterizzante di sperimentazione e commistione. Testori, al principio degli anni Settanta, affrontava sicuramente come drammaturgo (verrà poi l'uomo di scena nel senso più ampio del termine) direzioni diverse sia dal suo passato "viscontiano" (del resto imparagonabile dal punto di vista delle scelte linguistiche) sia dalla collocazione all'ombra di Gadda che, per puri motivi di "lombardità", Contini continuerà a richiamare,

7. Utilizzo qui l'analisi serrata (linguistica e con prospettive ontologiche) di Giorgio Agamben (2017, pp.89-112, in part. pp.103-106), con la distinzione, appunto, tra enunciati che "descrivono uno stato di cose esterno" ed enunciati che "attraverso il loro semplice proferimento, producono come un fatto ciò che significano", ovvero tra relazione apofantica (nel senso aristotelico) e comando. 
come luogo comune, dopo la prima inclusione nella rapida rassegna dei continuatori al tempo dell'intervento dedicato alla Cognizione del dolore, nel 1963.

Si rilegga dunque - mi limito a un solo prelievo - la battuta che apre proprio l'Ambleto, in cui il capocomico della scombinata troupe degli scarozzanti (insieme zitanes dell'underground provinciale!), che sta per entrare in scena nei panni di un improbabile principe di Danimarca, rivolge alcune istruzioni dietro le quinte a immaginari pittori di scena. Si noti: istruzioni per una "scena" che visivamente non apparirà agli spettatori:

(da fuori) Piu in dell'iscuro! Più in dell'iscuro! Rosso, sì. Ma rosso com'è rosso el sanguo dei zinghiali e dei porchi quando ce spaccheno in de su la gola!

Ha da esserci in dappertutto l'aria de un crotto! Ha da esserci l'aria d'un buso, d'un inferna!

Più ingravedate quelle nìgore! Più ingravedate e anca più incostrate! No! Sulla crose, no! Sulla crose, lassatela income è! Ultimi resti, frattaglie ultime et estreme della fede... (Ambleto entra) (Testori, 2003, p. 1147).

Testori, dunque, tornando all'esempio citato, mi sembra - passando dal tempo dell'Arialda a quello dell'Ambleto - piuttosto un vistoso "epigono" (termine che non implica nella presente ripresa giudizio negativo o sminuente, anzi, al contrario, trattandosi di una delle prove di gran lunga più significative del teatro italiano del secondo Novecento) del suo effettivo maestro, appunto Longhi, che non un seguace tra un decennio e l'altro del Gadda milanese, sia per insufficienza di densità espressionistico-dialettale nelle prove giovanili, teatrali e non, sia per un altrettanto forte caratterizzazione espressiva e di "violenza al sistema”, nel senso dell'espressione e dei contenuti, nella sua produzione di quegli anni Settanta, qui all'incrocio appunto con un lombardo immaginario, misto di elementi alti e arcaici, che ha certamente modelli o precedenti prossimi, come la lingua del Mistero buffo di Fo e dell'Armata Brancaleone. ${ }^{8}$

$\mathrm{Ma}$, si potrebbe sospettare a questo punto, si offre qui proprio un reinvestimento, diciamo "creativo", da parte del Testori maturo della prosa del precedente Testori critico d'arte e soprattutto del suo maestro. Contini avrebbe, ci sembra, potuto cogliere un'eccezionale convergenza dei suoi percorsi e non saprei se egli abbia semplicemente guardato distrattamente alle prove di Testori o se si sia volontariamente trattenuto al di qua di un tale riconoscimento, certo più facile per la nostra distanza temporale di osservatori. In questa straordinaria creazione, sulla pelle di Franco Parenti, mi sembra indubitabile che l'oltranza formale, e insieme contenutistica, si attuasse proprio attraverso la parodia, dove l'espressionismo è anche e precisamente parodia di quei procedimenti nei quali Contini indicava il nesso "rinnovamento del linguaggio novecentesco". Il fatto che tutto ciò sia posto qui sotto un segno metateatrale permette anche un rapporto che in qualche modo si può collegare a quella

8. Ho già richiamato in altra occasione la testimonianza di Mario Monicelli relativa al rilievo in tale costruzione del tono enfatico da tradizione da teatro "di prosa" di cui era portatore Vittorio Gassmann (quello degli Amleti italiani e degli eroi alfieriani) per la costruzione "comica", come un'evidente trasposizione parodistica. 
corrispondenza o inclusione dell' istanza del "discorso rappresentativo" nel "discorso referente", secondo appunto la descrizione continiana che abbiamo considerato. L'aggettivo "riflessa" che fondava la stessa categoria crociana di "letteratura dialettale", di principale e forte riferimento per Contini (ora D'Onghia ha convenientemente approfondito questo legame), postula evidentemente per il suo funzionamento un rapporto di rispecchiamento che si potrebbe utilmente collegare al doppio piano o al "doppio discorso" ora considerati: ogni riattraversamento metalinguistico, metateatrale o metalettico (nel senso del passaggio e dello scambio tra i livelli dell'autore, dei personaggi, degli spettatori e lettori) e di tutti i meta-che si vogliono, è qui attuato attraverso la parodia. Se, come abbiamo già detto, è ovvio supporre prima dell'Ambleto e del Macbetto il Fo del Mistero buffo, o il medioevo scalcinato dell'Armata Brancaleone e della sua straordinaria invenzione linguistica, ciò riguarda non solo le risorse di una libera e varia invenzione dialettale, ma dell'implicazione in essa di una simulazione di lingua "alta" e "arcaica" (e Brancaleone si pone infatti come un indubitabile referente, anche per esplicita allusione e citazione, nella messinscena originale dell'Ambleto). ${ }^{9}$ Non è un caso che i due registri qui rapidamente indicati caratterizzino altre rivisitazioni della tradizione che, riprendendo ma ricollocando una definizione ancora continiana in rapporto a Croce, ho in altri contributi definito "patrie temporali" caratterizzanti (come appunto il "barocco" napoletano).

$\mathrm{Ci}$ si può chiedere, in questa direzione, se ciò che a Contini appariva come positivamente "estremo" (anzi, residuo per ripresa) nella scrittura di Longhi, suo segno di forte sperimentalità primo-Novecentesca, non risultasse negli anni degli "ultimi esercizî" del grande critico involontariamente ridicolo o involontariamente parodistico. Credo che l'eccesso di "prosa d'arte" della scrittura longhiana - lo si apprezzi o lo si respinga, poco importa - mostri esattamente il suo reinvestimento, volontario o meno, nel Testori della Trilogia degli scarozzanti, dove una deliberata assunzione parodistica giunge fino ad attingere, proprio attraverso la deformazione, a un'altra e diversamente profonda "verità", a quella dimensione che l'autore definisce, nel suo intervento di poetica teatrale più rilevante, come il "ventre del teatro". Parodistica, posta anche la non proponibilità o la consunzione di altri modelli interpretativi, è la funzione che permette tale comprensione e riproposizione, dove Ambleto risulta tanto più "tragico" rispetto agli Amleti della convenzione del teatro di "prosa". Questa una via principale e specifica della scena italiana per il recupero e la reinvenzione della tradizione, impossibile a culture non provviste come quella italiana di ampia e stratificata storia linguistica, attraverso la dialettalità e il plurilinguismo, che si definiva proprio in quegli anni, con episodi che giungono alla profondità estrema e lacerante additata dalle grandi esperienze di punta della scena del Novecento, dove "doppi", "crudeltà", "pesti" si attingono per questa via: "comme ce lo conta Antonio Artiò", scriveva Roberto De Simone, in una significativa meditazione, ricorrente anch'essa, benché prefazione di libro, al

9. Disponibile su https://www.youtube.com/watch?v=QZ-bpxzT7RQ 
pastiche, in cui il riferimento esplicito al terreno del principale "rinnovamento" del teatro del primo Novecento si serve di un congruo abbassamento di tono. ${ }^{10}$ Luca D'Onghia - riattraversando l'intera tradizione del plurilinguismo teatrale italiano - ha significativamente proposto in un saggio fresco di stampa la definizione di "ritorno del superato linguistico" e anche, e precisamente, di "ritorno del represso linguistico", laddove tale scelta si colleghi a "contenuti" che possiamo dire censurati e repressi. ${ }^{11}$

5. Anche la questione dell'ecfrasi a teatro - oggetto di queste annotazioni offre a partire da tali considerazioni spunti per riflessioni ulteriori, guardando specificamente a questo panorama, come l'incipit dell'Ambleto, con gli ordini relativi al completamento di una "scena" che non si vedrà (almeno nell'attuazione originale) ha indicato con estrema efficacia.

Peraltro la stessa storia della "scenificazione" (qui interamente "d'autore") di Mistero buffo, in una scelta che risolve un testo inizialmente concepito per organico di compagnia e poi assunto nell'affabulazione solitaria, offre degli elementi rilevanti per un'osservazione ulteriore. Si vedano le didascalie, probabilmente all'inizio un residuo della "forma" originale, ma tuttavia sempre mantenute nelle varie edizioni del testo (o dei diversi "agglomerati" di pezzi che si fissano sotto questo titolo). Queste didascalie non sono infatti state eliminate nemmeno nella sistemazione in un volume complessivo, nel 2000, che fa posto anche a pezzi "impossibili", nella messa in pagina della pura simulazione onomatopeica del grammelot, come La fame dello Zanni), a dimostrare che non si tratta di puri residui. Se ne vedano un paio di esempi:

Sul fondo appare la Morte avvolta in un gran mantello nero. Un velo leggero lascia trasparire un viso "mortalmente pallido". [...] Il Matto volta le spalle alla Morte. Ė intento a contare le poche monete che gli sono rimaste.

Cinque uomini (gli Inchiovatori) che alla cintola tengono infilate mazze e lunghi chiodi stanno accingendosi a crocifiggere Cristo. La croce è già distesa sul terreno. Sul fondo, dietro a un lenzuolo steso, in controluce vediamo Gesù che, costretto dai soldati, si spoglia, mentre alcune donne appartate in un angolo del proscenio, seguono l'azione (Fo, 2000, 388 e 401).

Non siamo evidentemente di fronte a didascalie impossibili in rapporto alla loro realizzabilità, ma per l'estraneità della visualizzazione alla scelta

10. Nella pagina di premessa in Cappuccio (1997).

11. Si veda dal titolo allusivamente orlandiano Per una teoria della letteratura dialettale in Italia (si potrebbe, debitamente inteso, aggiungere l'aggettivo freudiana), in Brugnolo, Campeggiani \& Danti (2020, pp. 229-245). Questa proposta, beninteso, è parallela a quella del riconoscimento e della valorizzazione - dalla Veniexiana a Goldoni - di una "inclinazione alla 'realtà poco o per niente diffusa nella letteratura scritta in italiano" dell'uso del dialetto, che nulla ha di contrastivo o di "violenza al sistema", ovvero di espressionistico. Si vedano nel saggio le riflessioni sul "parametro quello relativo all'emersione di un represso tematico" e di un "ritorno del represso linguistico [che] sia stato in certi casi veicolo di un ritorno del represso tematico assai più forte e trasparente di quello che si dà per solito nei testi in lingua" (p. 241). 
monologica, interamente mimico-narrativa, in una prospettiva che possiamo appunto definire come "ecfrastica", laddove la "pittura in parole" definisce l'opera che non si dà nella sua concretezza di oggetto. Ma esse sono rivolte non all'immaginazione dello spettatore, altrimenti stimolata dalla potenza del "mimo", ma del lettore.

Gli esempi si potrebbero moltiplicare a volontà, e mi limito a notare che nella seconda delle due citazioni, la descrizione non si riferisca alla "storia" narrata sulla scena nuda dall'attore unico (al luogo della crocifissione di Cristo), ma al luogo di una (non attuata) "messa in scena" secondo i principi della finzione drammatico-rappresentativa: "in un angolo del proscenio", appunto, non del monte Golgota.

E, allargando il campo, viene da aggiungere che la storia teatrale di Fo risulta complessivamente segnata dalle sue attitudini di "artista", diplomato all'Accademia di belle arti di Brera, sia nell'inclusione delle immagini nel testo, sia della loro proiezione o esecuzione dal vivo, che conduce fino alla vera e propria deriva dell'attività degli ultimi anni (si permetta il giudizio netto e negativo, come sempre uguale e contrario a quello riservato alla fase alta della storia di Fo), in improbabili rifacimenti per sua mano di opere di grandi pittori, criticabili non per la loro libertà, ovviamente, ma per la sostanziale banalizzazione.

Questo e altri casi in cui la pittura dal vivo - dall'action painting alla più banale proiezione di immagini - si pone ben oltre la "realizzazione scenografica" come elemento fondamentale dello spettacolo rilanciano il tema dell'ecfrasi, nei suoi limiti di determinazione (dove lo spettatore vede ciò che sente raccontare) e in quelli, più specifici, di un'ecfrasi "performata" (torno a De Min e alla definizione di "ostensione" dell'immagine).

Terminerò dunque con un esempio che si pone - per tenere insieme le due tracce che ho fin qui provato ad intrecciare, partendo da Contini - sia sul terreno di una moderna "letteratura drammatica riflessa" (da giudicare in che senso e in che grado "espressionistica") sia su quello, precisamente, di un' "ecfrasi performata". Il testo da cui traggo la citazione di chiusura è uno dei più ragguardevoli e fortunati dal punto di vista della storia rappresentativa, mettendo in campo un'ulteriore "funzione", di quella "funzione Shakespeare" nella ricreazione dialettale che abbiamo coinvolto con le due prime prove della testoriana "trilogia degli scarozzanti". Essa rinvia all'altra, e certo principale, "patria", temporale e territoriale, di questa ampiamente significativa tradizione, ovviamente quella napoletana (e che implica, nel "dopo De Simone", quale principale punto di riferimento della Basile-Renaissance e del riferimento alla Napoli secentesca e "barocca", anche il grandissimo episodio conclusivo della carriera di Eduardo, con la traduzione e l'incisione su nastro della Tempesta di Shakespeare). Si tratta di Shakespea Re di Napoli di Ruggero Cappuccio, che nelle sue varie redazioni e revisioni, "preventive" e "consuntive" rispetto alle messinscena, si offre tra gli anni Novanta e Duemila come un esempio di grande significato. 
Se il testo non mi sembra possedere le valenze di "violenza al sistema" o di "ritorno del represso", esso risulta tanto più interessante nella dimensione del "superato" o del confronto o rispecchiamento nella tradizione antica, della "patria temporale" elettiva (la stessa in effetti della "letteratura dialettale riflessa" di Croce, che egli naturalmente pensava a partire da Napoli), che è insieme la città dei succhi espressionistici e la Napoli "gentile" del primato del dialetto. E infatti troviamo qui non solo intrecciati nell' elevazione del registro napoletano ma anche giustapposti il registro espressivo-popolare e quello dell'italiano, anche se connotato da tratti locali, come registro alto e nella sua impronta remota o "barocca" ampollosamente costruito (e qui, se si ritorna in senso lato alla "funzione Gadda" di Contini, si può rammentare come la sperimentazione del Pasticciaccio e della Cognizione, in questo caso "moderna", si affiancasse a quella, secondaria ma significativa, arcaica e "letteraria", del Primo libro delle favole).

L'invenzione di Cappuccio immagina dietro all'iniziale del nome (dibattutissimo) del dedicatario dei Sonetti di Shakespeare - W. sciolta come Will, inteso non già come diminutivo di William, ma tradotto alla lettera - un giovane cantimbanco napoletano di nome Desiderio, rapito dal Bardo passante per Napoli, dopo un incontro a una festa di corte in maschera, e portato a Londra, facendone l'interprete sulla scena di alcune sue figure femminili. Tutto ciò - nel colloquio di Desiderio col suo compare Zorastro e nei campioni di lettere che il testo ci offre - risulta indecidibile, se ricostruzione di quanto accaduto dopo il ritorno di Desiderio a Napoli o pura proiezione immaginativa.

Sarebbe interessante - anche e in particolare per la questione della didascalia - confrontare le redazioni del testo, del resto nella direzione precisamente sottolineata dall'autore stesso, nella riappropriazione dopo l'utilizzo scenico (in una forma definita "letteratura musicale a due voci" o, ancora, nuovo disegno "nella forma di otto memorie private"). La dichiarazione fa senz'altro al caso nostro: "L'abolizione delle didascalie insieme con lievi o radicali permutazioni interne al testo teatrale rispecchiano ulteriori evoluzioni del lavoro che l'autore compie da tempo ai margini e nel corpo della parola scritta e di quella recitata fecondando la doppia dimensione della lettura e dell'ascolto di un'opera teatrale intesa come addizione fascinatoria del teatro immaginabile o incarnato" (Cappuccio, 2002, p. VIII). ${ }^{12}$ Peraltro le didascalie della prima redazione offrirebbero un ottimo esempio per il discorso fatto più sopra, posta la loro non realizzazione, a proposito di una collocazione sul piano del "teatro immaginabile".

Ma veniamo senz'altro, nel registro dell'italiano alto-arcaico, al pezzo o monologo finale (intitolato "Frammento ottavo»), che mette proprio al centro un episodio particolarmente significativo di ecfrasi, posto che esso ha come referente un quadro - che dovrebbe contenere l'immagine di Desiderio fatta dipingere a Londra da Shakespeare (e dunque la prova della verità del suo racconto) -, che Zoroastro scopre dopo la sua morte, sollevando il drappo 
che lo nasconde. Qui l'ecfrasi non riguarda la descrizione di un'immagine che non si vede o che si potrebbe vedere, ma appunto una cornice che nulla rivela sotto il drappo rosso ("amplissima e rabbuiata stoffa colore del sangue", anche per continuare il riferimento al prima citato "rosso scuro" dell' Ambleto, rosso "com'è rosso el sanguo dei zinghiali e dei porchi quando ce spaccheno in de su la gola", e forse con un possibile richiamo a una "funzione Caravaggio", pertinente tanto alla dimensione barocco-lombarda che a quella barocconapoletana, diciamo delle Sette opere di misericordia).

Mi pare un'immagine bellissima per i percorsi dell'ecfrasi teatrale, nell'assenza della visione nel luogo in cui essa si attende per definizione - la scena - e nel potenziamento tanto più forte che deriva proprio dall'assenza dell'oggetto, per la questione dell' "immaginato" e dell' "incarnato". E si tratta anche, ovviamente, nell'alternanza e nel rispecchiamento di prevalente napoletano arcaico ed espressivo, diciamo da Basile o Cortese, e di italiano alto ed enfatico, di un preciso campione stilistico:

Et avendo riguardato a lungo Desiderio che si stava fermato e freddamente immobile, dentro la quieta pazzia de lu dolore mio, gli occhi miei propri si sorpresero ne lo fissare lo quadro grande, che esso pure immobile si stava, ricoperto da una amplissima e rabbuiata stoffa colore del sangue. E avvicinato che mi fui all'opera ancora così ammantata, ne la folle passione di volere contemplare lo quadro presso lo sembiante disfiorito de l'indimenticato mio compagno, principiai a discostare la rossa stoffa da la cornice dell'opera et quando nella terrificata curiosità che a me avvinceva l'avetti tutta intiera disvelata, scopersi come l'ornato ligneo perimetro non racchiudeva tela nessuna: imperocché, dentro di esso si apriva lo vuoto e lo vuoto solamente signorìa teneva, mentre io stesso unicamente vedevo lo corpo vivo e spirato, la sincera et materiale imagine di Desiderio morto e vivo in quello quadro per sempre. (Cappuccio, 2002, p. 49)

\section{Bibliografia}

Agamben, G. (2017). Che cos'è un comando? In Creazione ed anarchia. L'opera nell'età della religione capitalista, Vicenza, Neri Pozza.

Brugnolo, S., Campeggiani, I. \& Danti, L. (edd.) (2020). L'amorosa inchiesta. Studi di letteratura per Sergio Zatti. Firenze: Cesati.

Cappuccio, R. (2002). Shakespea Re di Napoli. Torino: Einaudi.

Contini, G. (1988). Ultimi esercizî ed elzeviri (1968-1987). Torino: Einaudi.

D'Onghia, L. (2020). Per l'espressionismo di Contini, in Studi di filologia offerti dagli allievi a Claudio Ciociola. Pisa: ETS.

De Min, S. (2013). Leggere le didascalie. Bologna: Archetipolibri.

De Min, S. (2016). Decapitare la Gorgone. Ostensione dell'immagine e della parola nel teatro di Anagoor. Corazzano (PI): Titivillus.

De Min, S. (2018). Ekphrasis in scena. Per una teoria della figurazione teatrale. MilanoUdine: Mimesis.

Dupont-Roc, R. \& Lallot, J. (edd.) (1980). Poetica. Paris: Seuil.

Fo, D. (2000). Teatro. Torino: Einaudi. 
Pirandello, L. (1986-2007). Maschere nude, a cura di A. d'Amico, con la collaborazione di A. Tinterri. Milano: Arnoldo Mondadori.

Testori, G. (2003). Opere, II (1965-1977), a cura di F. Panzeri. Milano: Bompiani. Vescovo, P. (2007). Entracte. Drammaturgia del tempo. Venezia: Marsilio.

Vescovo, P. (2011). Il tempo a Napoli. Durata temporale e rappresentazione. Venezia: Marsilio.

Vescovo, P. (2015a). "Nave pitoresca", in Marco Boschini. L'epopea della pittura italiana nell'Europa barocca, a cura di E. M. Dal Pozzolo. Treviso: Zel Edizioni.

Vescovo, P. (2015b). A viva voce. Percorsi del genere drammatico. Venezia: Marsilio.

Vescovo, P. (2020). L'incerto fine. La peste, la legge, il teatro.Venezia: Marsilio.

Zorzi, L. (1979). Storia dell'arte italiana, I. Questioni e metodi. Torino: Einaudi. 